14 Luger G F. Artificial intelligence: structures and strategies for complex problem solving. 5th ed. Edinburgh: Pearson Education Limited, 2005

15 Giarratano J C, Riley G D. Expert system: Principles and programming. 4th ed. Stamford: Thomson learning, 2006

16 Newman T R. Multiple objective fitness functions for cognitive radio adaptation. Doctoral Dissertation. Kansas: University of Kansas, 2008

17 Baldo N, Tamma B R, Manoj B S, et al. A neural network based cognitive controller for dynamic channel selection. In: Proceedings of the IEEE International Conference on Communications, 2009, Dresden. Washington DC: IEEE, 2009. 1-5

18 Zhu X, Liu Y, Weng W, et al. Channel sensing algorithm based on neural network for cognitive wireless mesh network. In: Proceedings of the IEEE International Conference on Wireless Communications, Networking and Mobile Computing, 2008, Dalian. Washington DC: IEEE, 2008. 1-4

19 Tumuluru V K, Wang P, Niyato D. A neural network based spectrum prediction for cognitive radio. In: Proceedings of the IEEE International Conference on Communications, 2010, Cape Town, South Africa. Washington DC: IEEE, 2010. 1-5

20 Baldo N, Zorzi M. Learning and adaptation in cognitive radios using neural networks. In: Proceedings of the IEEE Consumer Communications and Networking Conference, 2008, Las Vegas. Washington DC: IEEE, 2008. 998-1003

- 动 态.

\title{
心音身份识别: 一种生物特征识别新技术
}

生物特征识别作为身份认知方式具有独特的优势，它的 生物特征不像各种证件类持有物那样容易窃取丢失, 也不像 密码、口令那么容易遗忘或破解, 所以近年来在国际上被广 泛运用. 目前, 国内外研究领域中常见的生物特征识别有指 纹、手型、掌纹、虹膜、视网膜、耳廍、语音、步态以及手 部、面部模式等, 这些生物特征识别都是基于人体外部特征 的研究, 大部分容易被窃取和模拟. 如指纹识别系统就有网 上销售的指纹复制膜，号称“专蒙指纹考勤机”，专门用来复 制和替代指纹. 为了提高认知识别的准确性和安全性, 目前 已有不少科学家将目光投向人体内部的信息研究, 如心电 图、DNA、脑波信号等.

心音是一种来自于人体内部的自然信号, 这种信号在医 学中应用于听诊治疗有了较久的历史, 心音是人体内部最重 要的生理信号之一, 包括心房、心室、大血管、心血管及瓣 膜功能的生理信息. 人体的心音信号具备普遍性、独特性和 可采集性的生物特征, 而且是来自于人体内部的信号, 不容 易被模仿或复制, 因此针对心音信号的特征识别研究在科学 上与医学上是很有价值的.

心音身份识别作为生物特征识别的一个全新概念, 其中
有很多新的理论与技术问题, 南京邮电大学成谢锋教授等撰 写的“心音身份识别技术的研究”一文, 进一步详细解答了心 音识别的有关问题. 该文发表于《中国科学 信息科学》2012 年第 2 期上.

论文在生物特征识别的大背景下研究了一种全新的生物 识别技术一心音身份识别. 通过分析心音信号的主要特点, 提出了具体的心音预处理技术, 在消除听诊器类型和位置变 化所产生影响的同时, 利用心音子波族形象地表征了心音的 生理特性. 这为设计针对性强、信息变得比较简单的心音识 别系统奠定了基础. 另外文章还提出了 HS-LBFC 特征参数提 取、相似距离等行之有效的识别方法, 这使得计算代价变小, 识别率明显提高, 为心音身份识别的实际应用开辟了一个新 途径.

在当今以普通生物识别系统为主流的大环境下, 心音身 份识别技术是独树一帜的全新识别概念, 这一研究不但拓展 了生物识别新内容, 而且对人体心音的认识与利用也达到了 一个新的高度.

(本刊讯) 